\section{Labile Ernährungssituation bei fortgeschrittenen Kopf-Hals-Tumoren}

\begin{abstract}
Bedingt durch den Tumor selbst, Bestrahlungs- und Operationsfolgen kommt es bei Patienten mit Kopf-Hals-Tumoren oft zu einem erheblichen Gewichtsverlust. In einer retrospektiven Kohortenstudie an einem schwedischen Zentrum wurde untersucht, welche Faktoren den Ernährungsstatus dieser Patienten beeinflussen und ob es einem Zusammenhang zwischen Mangelernährung und Mortalität oder postoperativer Infektionsrate gibt.
\end{abstract}

D ie Gründe für einen Gewichtsverlust bei Patienten mit Kopf-Hals-Tumoren sind vielfältig: Schluckbeschwerden, Xerostomie, Mukositis, Appetitverlust, Schmerzen, Gebissprobleme sowie Ge-

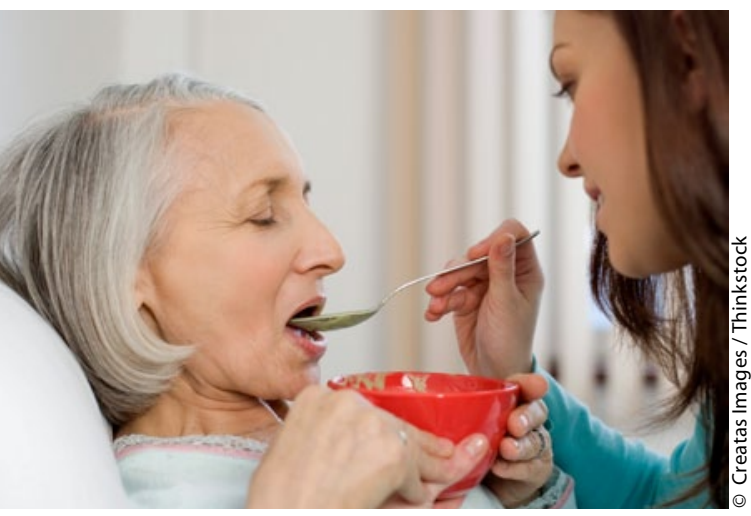

Der Ernährungszustand von Patienten mit Kopf-Hals-Tumoren sollte vor allem in den ersten sechs Monaten nach der Strahlentherapie genau überwacht werden. ruchs- und Geschmacksstörungen können dazu beitragen.

Einen Goldstandard für die Prävention und Behandlung des Gewichtsverlustes gibt es bisher noch nicht. Außerdem ist noch nicht endgültig geklärt, welchen Einfluss der Gewichtsverlust auf Überlebenschancen und Morbidität der Patienten hat.

Im Rahmen der Studie wurde der Ernährungszustand von 178 Patienten mit Kopf-Hals-Tumoren nach der Strahlentherapie über zwei Jahre in einer ambulanten Klinik regelmäßig kontrolliert. 73 Patienten litten an einem Oropharyngeal-Karzinom, 42 an einem Karzinom der Mundhöhle und 37 an einem Larynxkarzinom - der Rest verteilte sich auf weitere Tumorarten.

Der Höhepunkt des Gewichtsverlustes wurde sechs Monate nach der Strahlentherapie beobachtet. Insgesamt wurden 59\% der 157 erfolgreich therapierten $\mathrm{Pa}-$ tienten (kein Resttumor) zu irgendeinem
Zeitpunkt enteral über eine Sonde ernährt. Diese zeigten einen deutlich höheren Gewichtsverlust als Patienten, bei denen eine orale Ernährung möglich war $(13 \%$ vs. $6 \%)$. Am ausgeprägtesten war der Gewichtsverlust bei kombinierter Therapie (Operation und Bestrahlung) - obwohl hier $68 \%$ zwischenzeitlich über eine Sonde ernährt wurden.

Einziger unabhängiger Risikofaktor für einen ausgeprägten Gewichtsverlust war ein höheres Tumorstadium. Tumorlokalisation, Operation, Geschlecht oder Alter hatten dagegen keinen unabhängigen Einfluss. Es wurde kein Zusammenhang zwischen Gewichtsverlust und der Rate an postoperativen Infektionen festgestellt.

Fazit: Die Studie zeigt, dass es bei einem Großteil der Patienten mit Kopf-Hals-Tumoren trotz ambulanter Überwachung des Ernährungszustandes zu einem deutlichen Gewichtsverlust kommt. Auch eine enterale Sondenernährung scheint den Gewichtsverlust nicht vollständig aufhalten zu können. Die Überwachung des Ernährungszustandes ist insbesondere in den ersten sechs Monaten nach der Strahlentherapie und bei Patienten mit höheren Tumorstadien erforderlich, schreiben die Autoren.

Maria Weiß

Ehrsson YT et al; Nutritional surveillance and weight loss in head and neck cancer patients; Support Care Cancer 2012: 20: 757-765

\section{Nach welcher Zeit sollte der Pricktest abgelesen werden?}

Ein Pricktest ist in der allergologischen Diagnostik ein preiswertes, erprobtes und bewährtes Verfahren. Routinemäßig wird er üblicherweise nach 15 bis 20 Minuten abgelesen. Unklar ist aber weithin, wie stark die Schwankungen sein können, um trotzdem verlässliche Werte zu bekommen.

$\mathrm{D}$ ie Frage nach dem idealen Zeitpunkt zur Auswertung eines Pricktests konnte nun ein amerikanisches Team um Sara Seibert vom College of Medicine der Penn State University in Pennsylvania klären: Dazu führten sie bei 57 Patienten in einer prospektiven Studie jeweils Pricktests mit unterschiedlichen AeroAllergenen durch und maßen dann nach 10, 20, 30 und 40 min die Testquaddel und das umgebende Erythem. Sie verglichen dann die Quaddeln und Erytheme, die nach der üblichen 20-min-Wartezeit auftraten, mit denen nach 10, 30 und 40 min.

In der statistischen Auswertung sahen sie eine enge Korrelation der Flächen bei der 20- und 30-minütigen Ablesung, jedoch eine deutlich schwächere zu den Resultaten nach 10 bzw. 40 min. Bei noch kürzerer Ablesezeit (weniger als 10 min) und auch bei längerem Zuwarten als 40 min. sank die Verlässlichkeit.

Fazit: Für das Ablesen des Pricktests ist die traditionelle Zeitspanne von 15 bis 20 min nach dem Tests am besten geeignet; sollten aber in der Praxis widrige Umstände den empfohlenen Ablesezeitpunkt verhindern, kann auch im Zeitraum von 10 bis 40 min nach dem Piks noch ein verlässliches Ergebnis erzielt werden. Dieses Resultat gibt den Praxen mehr Flexibilität. Zeiten unter 10 und über 40 min beinhalten das Risiko einer deutlich abnehmenden Qualität. Dr. Ulrich Mutschler

Seibert SM et al. Reliability of skin test results when read at different time points. Allergy Asthma Proc 2011; 32: 203-5 